\title{
Zugriff auf das Recht der Europäischen Menschenrechtskonvention: \\ Eine Analyse der Zugangsbedingungen zur judikativen Rechtserzeugung Straßburgs
}

Reto Walther

Abstract: Dieser Beitrag diskutiert das judicial law-making des Europäischen Gerichtshofs für Menschenrechte (EGMR) anhand demokratie- und gewaltenteilungstheoretischer Argumente. Dem EGMR wird heute ein verfassungsgerichtsähnlicher Status zuteil. Sein großer Einfluss auf die staatlichen Verfassungsordnungen wird indes kritisiert. Diese Einflussnabme geht insbesondere auf die judikative Rechtserzeugung des EGMR zurück. Der Beitrag argumentiert, dass dieses judicial law-making, obwobl es unvermeidbar ist, demokratie- und gewaltenteilungstheoretische Probleme aufwirft. Diese Probleme sollten möglichst vermindert werden, indem das Verfahren vor dem EGMR in puncto Repräsentativität und Partizipativität, Zugangs- und Zulässigkeitsbedingungen sowie inhaltlicher Offenheit verbessert wird. Der Beitrag skizziert einige Vorschläge dazu. Er endet mit Überlegungen, warum der EGMR trotz den diskutierten Problemen eine demokratisch legitime Rolle spielt.

\section{Einleitung}

Der Europäische Gerichtshof für Menschenrechte genießt einen verfassungsgerichtsähnlichen Status. ${ }^{1}$ Diese „Adelung“ geht auf die Entwicklung zurück, die die Vorgängerinstitutionen des Gerichtshofs in den 1970er-

1 Bates, Evolution of the ECHR, 2010, S. 225; Keller/Kühne, ZaöRV (2016), 245; Madsen, Law \& Contem Prob (2016), 141 (166-167); Marti, Framing a Convention Community, Diss. Zürich, 2018 (CUP 2021), Kap. 3; Pollinico, in: Repetto (Hrsg.), Constitutional Relevance of the ECHR, 2013, S. 99 (100); Rosenfeld, EJIL (2014), 177 (193-194); Sadurski, HRLR (2009), 397 (449-450); Stone Sweet, RTDH (2009), 923 (944). 
und 1980er-Jahren durchlaufen ${ }^{2}$ und angetrieben haben. ${ }^{3}$ Dabei hat sich das Kräfteverhältnis zwischen dem Gerichtshof und den Mitgliedsstaaten der Europäischen Menschenrechtskonvention ${ }^{4}$ deutlich zugunsten des Gerichtshofs verschoben. ${ }^{5}$ Die Staaten haben dies lange unterstützt. ${ }^{6}$ Doch seit etwa zehn Jahren wird Kritik geäußert. Letzteres ist erkennbar an der Verankerung des Subsidiaritätsprinzips und der "margin of appreciation“ in der Konventionspräambel. ${ }^{7}$ Ersterem Prinzip zufolge kommt dem Gerichtshof lediglich eine subsidiäre Rolle zu; zweiteres gewährt den Staaten einen Ermessensspielraum in der innerstaatlichen Umsetzung der Konvention. ${ }^{8}$ Opposition kommt auch vonseiten nationaler Gerichte, die sich gegen den Gerichtshof auflehnen, ${ }^{9}$ und wissenschaftlichen Kritikern. ${ }^{10}$

Für den vorliegenden Beitrag lässt sich die Kritik zusammenfassen in Missmut über einen Gerichtshof, der zu mächtig wurde und zu stark in den Einflussbereich der Staaten eindringt, ${ }^{11}$ der seine Kompetenzen ausdehnt und der den Staaten aufgrund seines Verständnisses der Konvention als „living instrument" ${ }^{\text {"12 }}$ in unvorhersehbarer und teils willkürlich anmutender Weise immer neue Konventionsverletzungen anlastet und

2 Bates, Evolution (Fn. 1), Kap. 7-8; Madsen, in: Christoffersen/Madsen (Hrsg.), ECtHR Between Law and Politics, 2011, S. 43 (51-54); siehe auch Madsen, La genèse de l'Europe des droits de l'homme, 2011; Simpson, Human Rights and the End of Empire, 2001.

3 EGMR, Urt. v. 21.2.1975 - Nr. 4451/70, A-18 - Golder; EGMR, Urt. v. 6.9.1978 Nr. 5029/71, A-28 - Klass; EGMR, Urt. v. 8.6.1976 - Nr. 5100/71 u.a., A-22 - Engel; EGMR, Urt. v. 26.4.1979 - Nr. 6538/74, A-30 - Sunday Times; EGMR, Urt. v. 7.12.1976 - Nr.5493/72, A-24 - Handyside; EGMR, Urt. v. 25.4.1978 Nr. 5856/72, A-26 - Tyrer; EGMR, Urt. v. 13.6.1979 - Nr. 6833/74, A-31 - Marckx; EGMR, Urt. v. 9.10.1979 - Nr. 6289/73, A-32 - Airey.

4 Konvention zum Schutze der Menschenrechte und Grundfreiheiten (EMRK), 4.11.1950, in der Fassung der Protokolle Nr. 11 und 14 (CETS Nr. 005).

5 Christoffersen/Madsen, in: Christoffersen/Madsen (Hrsg.), ECtHR Between Law and Politics, 2013, S. 230 (236-239).

6 Änderungsprotokolle Nr. 11, 11.5.1994, und Nr. 14, 13.5.2004.

7 Art. 1 Zusatzprotokoll Nr. 15, 24.6.2013, (CETS Nr. 213); kürzlich auch Kopenhagen Deklaration, Ministertreffen, 12.-13.4.2018.

8 Explanatory Report to Protocol No. 15, 24.6.2013, Rn.9.

9 Keller/Walther, GCYBILJ (2020), 83 (86-96).

10 Bspw. zum Schrifttum in der Schweiz Keller/Walther, in: Breuer (Hrsg.), Principled Resistance, 2019, S. 161 (175-184).

11 Vgl. von Bogdandy, In the Name of the European Club of Liberal Democracies, $<$ https://cutt.ly/VkNVyfp>, abgerufen am: 31.3.2021.

12 EGMR, Tyrer (Fn. 3), Rn. 31; Dörr, in: Abi-Saab u. a. (Hrsg.), Evolutionary Interpretation, 2019, S. 115 (115-121). 
stets neue Menschenrechtspflichten auferlegt. ${ }^{13}$ Der Beitrag diskutiert den Vorwurf des illegitimen judicial law-making. ${ }^{14}$ Er knüpft am Unbehagen darüber an, dass der Gerichtshof zunehmend politische Fragen zu Rechtsfragen mache und sie so unter seine Kontrolle bringe. ${ }^{15}$

In einem ersten Schritt wird judicial law-making theoretisch eingeführt (II), bevor das law-making des EGMR exemplarisch aufgezeigt wird (III). Abschnitt IV diskutiert das Straßburger judical law-making als verfassungsund gewaltenteilungstheoretisches Problem. Nach Möllers Gewaltenteilungstheorie ${ }^{16}$ besteht das Problem darin, dass gerichtliche Institutionen und Verfahren anders als politische Gesetzgebungsprozesse grundsätzlich nicht darauf ausgerichtet sind, rechtsetzerisch tätig zu sein. In einem vierten Schritt werden Elemente des Verfahrens vor dem Gerichtshof erörtert (V), die für die demokratische Legitimation des Straßburger Richterrechts besonders problematisch sind. Abschnitt VI reflektiert Korrektive. Der Schlussabschnitt deutet Argumente an, die das identifizierte Problem relativieren.

\section{Judicial law-making: Theorie}

\section{Grundlagen}

Gerichtliche Entscheide, die als judicial law-making zu qualifizieren sind, zeichnen sich dadurch aus, dass sie sich in die Zukunft sowie an einen Adressatenkreis richten, der über die Streitparteien hinausgeht und potenziell alle Rechtsunterworfenen umfasst, materiell-rechtlich unterbestimmt also ergebnisoffen bzw. keiner kognitiv richtigen Lösung zugänglich sind und das Ziel verfolgen, die normativen Erwartungen des Adressatenkreises zu verändern, also rechtliche Normativität, die die Adressaten in ihrer Freiheit betrifft, zu erzeugen. ${ }^{17}$

13 Bspw. Seiler, ZBJV (2014), 265 (351-368); Seiler, in: Zufferey/Dubey/Previtali (Hrsg.), L'homme et son droit, 2011, S. 511 (523).

14 Der Anglizismus wird mangels adäquater Übersetzung beibehalten. Siehe von Bogdandy/Venzke, in: von Bogdandy/Venzke (Hrsg.), International Judicial Lawmaking, 2012, S. 3 (14).

15 Bspw. Sumption, The Limits of Law, 27th Sultan Azlan Shah Lecture, 20.11.2013, S. 6; Keller/Walther, Resistance (Fn. 10), S. 183 m. w. H.

16 Möllers, The Three Branches, 2013, Kap. 2.

17 Eingehend von Bogdandy/Venzke, Beyond Dispute (Fn. 14), S. 9-20. 
Mit Möllers lässt sich politisches (gesetzgeberisches) von gerichtlichem (adjudikativem) Entscheiden prototypisch wie folgt unterscheiden: Erstens ist der Gerichtsentscheid personal wie sachverhaltlich individualisiert. Im Fall des Gerichtshofs betrifft er ein von der Konvention garantiertes Freiheitsrecht eines bestimmten Beschwerdeführers in einer konkreten Situation. Politische Entscheide hingegen richten sich potenziell an alle und determinieren "general spheres of freedom and obligation". ${ }^{18}$ Zweitens erfolgt der Gerichtsentscheid retrospektiv, während der politische Entscheid prospektiv wirkt. ${ }^{19}$ Drittens ergeht der Gerichtsentscheid gestützt auf materielles Recht, das die strittige Situation materiell-rechtlich bestimmt, das also die strittige Freiheits- oder Interessensphäre autoritativ abgrenzt. ${ }^{20} \mathrm{Um}$ gekehrt wird der politische Entscheid von Verfahrensrecht geleitet, das darauf ausgelegt ist, den materiellen Entscheid - die zu bestimmende Politik („policy“) - nicht zu determinieren: Das gesetzgeberische Verfahren zielt auf die kontinuierliche Offenhaltung der Möglichkeit ab, eine policy zu revidieren. ${ }^{21}$ Der Gerichtshof ist demnach in dem Maße in law-making involviert, in dem sein Entscheiden prospektive Wirkung auf die Rechte und Pflichten potenziell aller Konventionsstaaten und der sich in ihrer Jurisdiktion befindlichen Personen ${ }^{22}$ hat.

\section{Konventionsrechtliche Eigenheiten}

Dieser scheinbar simple Befund vereinfacht einige Komplexitäten des Konventionssystems. Erstens haben die Urteile des Gerichtshofs weder erga omnes inter partes- ${ }^{23}$ noch formal präjudizielle ${ }^{24}$ Wirkung. Sogar das res interpretata-Prinzip hat der Gerichtshof nie wiederholt und explizit bestätigt. ${ }^{25}$ Doch sprechen Praxis und Systemlogik ${ }^{26}$ für die Annahme eines

18 Möllers, Branches (Fn. 16), S. 79.

19 Möllers, Branches (Fn. 16), S. 79.

20 Das heißt nicht, dass Recht vollständig determiniert ist. Siehe Möllers, Branches (Fn. 16), S. 83-84.

21 Möllers, Branches (Fn. 16), S. 79-80.

22 Art. 1 EMRK.

23 Art. 46 EMRK; Marti, Community (Fn.1), S.230; Villiger, Handbuch EMRK, 3. Aufl., 2020, Rn. 232.

24 EGMR, Urt. (GK) v. 11.7.2002 - Nr. 28957/95, ECHR 2002-VI - Goodwin, Rn. 74; Mowbray, HRLR (2009), 179 (180-187).

25 Singulär: EGMR, Urt. v. 9.6.2009 - Nr. 33401/02, ECHR 2009 - Opuz, Rn. 163; siehe auch Marti, Community (Fn. 1), S. 226.

26 Insb. Art. 1, 19, 32 EMRK. 
„legally binding effect of the generalizable parts“ der Urteile des Gerichtshofs. ${ }^{27}$ Zweitens kommt den Konventionsstaaten in der Urteilsumsetzung regelmäßig ein Ermessensspielraum zu. ${ }^{28}$ Drittens fordert das Konventionsrecht weder seinen Vorrang ${ }^{29}$ noch seine unmittelbare Wirkung oder direkte Anwendbarkeit ${ }^{30}$ im nationalen Recht, allenfalls aber die konventionskonforme Auslegung des innerstaatlichen Rechts. ${ }^{31}$ All dies scheint den Einfluss der Konventionsauslegung durch den Gerichtshof insofern zu relativieren, als es Raum für eine vermittelnde Übertragung des Konventionsrechts ins nationale Recht lässt.

Die innerstaatliche Perspektive qualifiziert diese Feststellung allerdings wieder. Das richterrechtlich entwickelte Konventionsrecht stellt in allen Mitgliedstaaten einen direkt anwendbaren ${ }^{32}$ integralen Bestandteil des nationalen Rechts dar, der letzterem (de facto) regelmäßig vorgeht. ${ }^{33} \mathrm{Zudem}$ hat der Gerichtshof seinen Einfluss im Bereich der Urteilsumsetzung stark ausgebaut $^{34}$ und das diesbezügliche Verfahren vor dem Ministerkomitee wurde partiell verrechtlicht und entpolitisiert. ${ }^{35}$

Schließlich gilt es, den „Inkrementalismus“ des Gerichthofs zu bedenken. Gemeint ist seine Praxis, die Konvention schrittweise (kasuistisch) zu entwickeln, so dass generell-abstrakt anwendbare Standards über viele Urteile hinweg entstehen. Dies erlaubt es dem Gerichtshof, Reaktionen der Staaten abzuwarten und in seine Konventionsentwicklung aufzunehmen. Dennoch verdichtet sich die Rechtsprechung an einem gewissen Punkt so weitgehend, dass den Staaten höchstens noch kleine Regelungsspielräume

27 Vgl. Marti, Community (Fn. 1), S. 240.

28 Vgl. unten Text zu Fn. 43 ff.

29 Vgl. aber Art. 27 Wiener Übereinkommen über das Recht der Verträge, 23.5.1969 (1155 UNTS 331); EGMR, Urt. (GK) v. 30.1.1998 - Nr. 19392/92, Reports 1998-I United Communist Party, Rn. 30.

30 Indirekt Art. 13 EMRK; bspw. EGMR, Urt. (GK) v. 10.7.2020 - Nr. 310/15 - Mugemangango, Rn. 130. Unklar EGMR, Urt. (GK) v. 29.3.2006 - Nr. 36813/97, ECHR 2006-V - Scordino (Nr. 1), Rn. 239; Marti, Community (Fn. 1), S. 265-267.

31 Marti, Community (Fn. 1), S. 268-272, gestützt auf EGMR, Scordino (Fn. 30).

32 Zum „de facto invalidating effect“ Besson, in: Popelier/van de Heyning/van Nuffel (Hrsg.), Human Rights Protection in the European Legal Order, 2011, S. 97 (112).

33 Stone Sweet, GlobCon (2012), 53 (84-85); Besson, Supranational (Fn. 32), S. 105 106; ferner Marti, Community (Fn. 1), S. 282-299; Martinico, EJIL (2012), 401.

34 Marti, Community (Fn. 1), S. 156-179.

35 Çali/Koch, HRLR (2014), 301. Den Nuancen und Schwierigkeiten der Integration des Konventionsrechts ins Recht der Mitgliedstaaten, und insbesondere den Problemen mit der Urteilsumsetzung, kann in diesem Rahmen leider nicht weiter Rechnung getragen werden. 
verbleiben. ${ }^{36}$ Außerdem kommt auch diese Praxis nicht ohne Leiturteile, die einen Wendepunkt in der Rechtsprechung markieren, aus. ${ }^{37}$

\section{Die Unvermeidbarkeit von judicial law-making}

Insgesamt kann das judicial law-making des Gerichtshofs nicht mit legislativer Rechtsetzung gleichgesetzt werden. Trotzdem weist der konsolidierte Effekt der Straßburger Praxis die zentralen Merkmale von Rechtsetzung auf. Einerseits ist dies aufgrund konventionsrechtlicher Eigenheiten anders, möglicherweise schwächer, ausgeprägt als im innerstaatlichen Kontext. Wie die folgenden Ausführungen zeigen werden, ist die Problematik andererseits jedoch verschärft aufgrund der Mannigfaltigkeit des Straßburger judicial law-making und der fehlenden Einbettung des Gerichtshofs in die politischen Prozesse einer demokratischen Verfassungsordnung.

Wie Marti feststellt, ist das Richterrecht des Gerichtshofs „particularly extensive both in quality and quantity“.38 Es schränkt den politischen Spielraum der Staaten je für sich wie auch kollektiv als „masters of the treaty" erheblich ein. Letzteres zeigt sich zunächst in der prozeduralen und institutionellen Ordnung des Konventionssystems. Beispielsweise erwähnt die Konvention das Piloturteilsverfahren nicht. ${ }^{39}$ Die Verfahrensregeln finden sich in Rule 61 der Rules of Court, ${ }^{40}$ die der Gerichtshof selbst erlässt. ${ }^{41}$ Dabei handelt es sich um judicial law-making einer konventionsändernden Dimension. ${ }^{42}$ Auch die Kompetenz sogenannter „Artikel 46-Urteile" oder "Quasi-Piloturteile" hat sich der Gerichtshof selbst gegeben. ${ }^{43}$ In Abweichung der grundsätzlich deklaratorischen ${ }^{44}$ Wirkung der Urteile des Gerichtshofs ${ }^{45}$ enthalten letztere Empfehlungen und in seltenen Fäl-

36 Gerards, HRLR (2018), 495 (506-512).

37 Bspw. EGMR, Goodwin (Fn. 24), Rn. 73, 93.

38 Marti, Community (Fn. 1), 192.

39 Vgl. aber Resolution of the Committee of Ministers, 12.5.2004, 114th Sess, Res(2004)3; Explanatory Report to Protocol No. 14, 13.5.2004, Rn. 16; Harmsen, in: Morison/McEvoy/Anthony (Hrsg.), Judges, Transition, and Human Rights, 2007, S. $33(45-47)$.

40 RC, 1.1.2020.

41 Fyrnys, in: von Bogdandy/Venzke (Hrsg.), International Judicial Lawmaking, 2012, S. $329(339-345)$.

42 Fyrnys, Pilot (Fn. 41), S. 348-349.

43 Mowbray, HRLR (2017), 451 (452-457).

44 Vgl. aber Art. 41 EMRK.

45 EGMR, Marckx (Fn. 3), Rn. 58. 
len Anordnungen im operativen Teil ${ }^{46}$ bezüglich Maßnahmen, die der betroffene Staat umsetzen muss, um das Urteil zu befolgen. ${ }^{47}$ Wenn diese Maßnahmen genereller Natur sind, also nicht nur die Wiedergutmachung des Einzelfalls betreffen, ${ }^{48}$ haben auch sie law-making Charakter, denn sie sind nicht retrospektiv-kompensatorischer, sondern prospektiv-präventiver $^{49}$ Natur. ${ }^{50}$

Im Mittelpunkt steht jedoch das judicial law-making des Gerichtshofs im Bereich der Konventionsgarantien. Leiturteile des Gerichtshofs, verstanden als Urteile, die den Konventionsrechten und den mit ihnen korrelierenden Staatspflichten neuen bzw. anderen Gehalt geben, weisen grundsätzlich immer ein Moment von judicial law-making auf. Dies folgt aus der triangulären Struktur von Grund- bzw. Menschenrechten. ${ }^{51}$ Gemeint ist die Triade Recht-Rechtsträger-Grundrechtsverpflichteter, wobei letzterer zwar der Staat ist, dieser aber die Mitglieder der politischen Gemeinschaft verkörpert. Wenn der Staat, der letztlich dem Wohl der Einzelnen dient, Grundrechte garantiert, verwendet er darauf Ressourcen, die nicht für andere ebenfalls im Interesse von Einzelnen stehende Zwecke verfügbar sind. Was dem einen geschützte Freiheits- oder Interessenssphäre ist, ist des anderen Verbot oder Pflicht. ${ }^{52}$ Die Bestimmung des Schutzgehalts eines Grundrechts hat kurzum immer eine wertende objektive Dimension, die potenzielle alle betrifft. ${ }^{53}$

Die aufgrund des Rechtssicherheitsbedürfnisses unabdingbare Präzedenzwirkung von Leitentscheiden bedeutet sodann, dass Leiturteile grund-

46 Erstmals EGMR, Urt. (GK) v. 8.4.2004 - Nr. 71503/01, Reports 2004-II - Assanidze, Pt. 14.

47 Jährliche Übersicht bei Committee of Ministers, Supervision of the Execution of Judgments and Decisions: Annual Report, <http://bit.ly/3iWMThh>, abgerufen am: 31.3.2021.

48 Colandrea, HRLR (2007), 396 (397-398).

49 Colandrea, HRLR (2007), 396 (408-409); Marti, Community (Fn. 1), S. 168-169.

50 Die Grenze ist oft unscharf. Bspw. EGMR, Urt. v. 26.3.2013 - Nr. 21794/08, ECHR 2013 - Zorica Jovanović, Rn. 92 und Pt. 6.

51 Bellamy, Political Constitutionalism, 2007, S. 30; Daly, in: Herlin-Karnell/Klatt/ Morales Zúniga (Hrsg.), Constitutionalism Justified, 2019, S. 197 (203-204); Finnis, Natural Law and Natural Rights, 2. Aufl., 2011, 200-202, 218-219.

52 Vgl. Bentham, in: Bowring (Hrsg.), Works of Jeremy Bentham, Bd. 2, 1843 [1796], 489 (503).

53 Vgl. BverfG, Urt. v. 15.1.1958 - 1 BvR 400/51, BVerfGE 7, 198 - Lüth, Rn. 25; Habermas, Faktizität und Geltung, 4. Aufl., 1994, S. 301-303; Lepsius, in: Jestaedt u. a. (Hrsg.), Das entgrenzte Gericht, 2011, S.159; Loughlin, Idea of Public Law, 2004, S. 127. 
sätzlich prospektiv wirken. ${ }^{54}$ Schließlich ist klar, dass die Interpretation von Bestimmungen - erst recht von äußerst abstrakten, quasi-verfassungsmäßigen Prinzipien wie den Konventionsgarantien - kein determinierter Kognitionsprozess des „Findens“ oder „Erkennens“ des „richtigen“ Verständnisses darstellt, sondern ein großes Moment an schöpferischer Tätigkeit beinhaltet. ${ }^{55}$

Mithin kann der Gerichtshof gar nicht anders, als Richterrecht zu setzen. Sein Mandat impliziert de facto ein "mandat de légiférer“. ${ }^{56}$ Dazu passt die Beobachtung, dass er „often behaves more as a general and prospective lawmaker than as a judge whose reach is primarily particular and retrospective". ${ }^{57}$ Inwiefern dieser Befund aus einer normativen, legitimationstheoretischen Perspektive problematisch ist, zeigt der nächste Abschnitt. Vorher lohnt es sich, die wichtigsten Fallgruppen dieses Phänomens kurz darzustellen.

\section{Judicial law-making: Praxis}

Eine erste Gruppe bilden Fälle konfligierender Konventionsrechte, also Fälle, denen ein Sachverhalt zugrunde liegt, in dem das vom Beschwerdeführer geltend gemachte Recht geradezu die Kehrseite des Rechts ist, zu dessen Schutz der betroffene Staat das Recht des Beschwerdeführers eingeschränkt hat. ${ }^{58}$ Das klassische Beispiel solcher „multipolarer“ Grundrechtskonstellationen sind Fälle zu Artikel 8- bzw. zu Artikel 10, die einerseits die Privatsphäre und andererseits die Pressefreiheit tangieren. ${ }^{59}$ Die Konvention äußert sich nicht zur Lösung solcher Konflikte. Deren Auflösung

54 EGMR, Urt. (PV) v. 18.1.978 - Nr. 5310/71, A-25 - Irland/UK, Rn. 154; EGMR, Urt. v. 7.1.2010 - Nr. 25965/04, ECHR 2010 - Rantsev, Rn. 197; CDDH, Report on the Longer-Term Future of the System of the ECHR, 11.12.2015, CDDH(2015)R84 Addendum I, Rn. 37; Árnardóttir, EJIL (2017), 819 (823-828); Bodnar, in: Haeck/Brems (Hrsg.), Human Rights and Civil Liberties, 2014, S. 223; Besson, in: Besson (Hrsg.), ECtHR After Protocol 14, 2011, S. 123 (134-135).

55 von Bogdandy/Venzke, Beyond Dispute (Fn.14), S.9-12; vgl. schon Kelsen, VVDStRL (1929), 30 (30-32).

56 Art. 19 EMRK; bspw. EGMR, Urt. v. 24.7.2003 - Nr. 40016/98, ECHR 2003-IX Karner, Rn. 26.

57 Keller/Stone Sweet, in: Keller/Stone Sweet (Hrsg.), Europe of Rights, 2008, S. 677 (703).

58 Eingehend Smet/Brems (Hrsg.), When Human Rights Clash, 2017.

59 NußBberger, in: Seibert-Fohr/Villiger (Hrsg.), Judgments of the ECtHR, 2014, S. 165 (172). 
steht also im Ermessen des Gerichtshofs und das Urteil betrifft die rechtliche Ausgestaltung von Freiheit nicht am Verfahren beteiligter Privater, gleich wer obsiegt. So hat der EGMR beispielsweise in der von HannoverSaga das Verhältnis zwischen Presse und Prominenten neu bestimmt. ${ }^{60}$ Aufgrund der triadischen Struktur von Grundrechten können indes alle Menschenrechtsfälle als „multipolar" rekonstruiert werden. ${ }^{61}$

Eine zweite Gruppe bildet die Rechtsprechung zu prozeduralen Rechten. Der Gerichtshof hat zunehmend konkrete Standards betreffend die Organisation der staatlichen Justizsysteme erarbeitet, und zwar entgegen seinen Bekräftigungen, ${ }^{62}$ dass es nicht seine Aufgabe sei, die Varietät der europäischen Justizsysteme zu vereinheitlichen. ${ }^{63}$ Kosař hat diese Praxis eingehend analysiert. ${ }^{64}$ In seinem Beitrag mit Lixinski werden als illustrative Fälle genannt: ${ }^{65}$ Frankreichs Abschaffung der avocats généraux des Conseil d'État, ${ }^{66}$ die Aufgabe der Military Security Courts in der Türkei ${ }^{67}$ und der Constitutional Reform Act 2005 betreffend die Gerichtsorganisation im Vereinigten Königreich. ${ }^{68}$ Auch die Schweiz hat ihr Verfahrensrecht aufgrund der Straßburger Rechtsprechung umfassend revidiert. ${ }^{69}$ Insgesamt hat der Gerichtshof großen Einfluss auf die institutionelle Justizordnung der Staaten. Kosař/Lixinski kommen zum Schluss, dass er das legislative Rechtsetzungsprimat konkurriere, insbesondere weil er im Rahmen von Beschwerden von nationalen Gerichtsmitgliedern auch nicht-prozedurale Konventionsrechte nutze, um die Gerichtsorganisation umfassend mitzubestimmen. Das habe zur Folge, dass er nicht nur das innerstaatliche horizontale Gewaltenteilungsverhältnis, sondern auch die Organisation,

60 Vgl. BverfG, Beschl. v. 15.12.1999 - 1 BvR 653/96, BVerfGE 101, 361 - Caroline von Monaco II; EGMR, Urt. v. 24.6.2004 - Nr. 59320/00, Reports 2004-VI - von Hannover; Nußberger, Subsidiarity (Fn. 59), S. 175-178.

61 Siehe Nußberger, Subsidiarity (Fn. 59), S. 173.

62 Ulfstein, IJHR (2020), 917 (920).

63 Bspw. EGMR, Urt. (GK) v. 27.5.2010 - Nr. 13279/05 - Şahin, Rn. 68.

64 Kosař, in: Wind (Hrsg.), International Courts and Domestic Politics, 2018, S. 71; Kosař, Utrecht L Rev (2017), 112; Kosař/Lixinski, AJIL (2015), 713.

65 Kosař/Lixinski, AJIL (2015), 713 (715-716).

66 EGMR, Urt. (GK) v. 12.4.2006 - Nr. 58675/00, Reports 2006-VI - Martinie; EGMR, Urt. (GK) - Nr. 39594/98, Reports 2001-VI - Kress.

67 Siehe insb. EGMR, Urt. (GK) v. 9.6.1998 - Nr. 22678/93, Reports 1998-IV - Incal; EGMR, Urt. v. 28.10.1998 - Nr. 19601/92 , Reports 1998-VII - Çıraklar; EGMR, Urt. v. 25.9.2001 - Nr. 29279/95, Reports 2001-IX - Şahiner.

68 Woodhouse, ICON (2007), 153 (154) m. H. a. EGMR, Urt. v. 8.2.2000 Nr. 28488/95, Reports 2000-II - McGonnell; EGMR, Urt. v. 28.9.1995 Nr. 14570/89, A-326 - Procola.

69 Wegweisend EGMR, Urt. (PV) v. 29.4.1988 - Nr. 10328/83, A-132 - Belilos. 
insbesondere die Hierarchie innerhalb, der staatlichen Judikativen beeinflusse. ${ }^{70}$ Kosar folgert: „the Strasbourg Court has become a key player in domestic judicial politics“. ${ }^{71}$

Eine dritte Gruppe sind Fälle positiver Staatspflichten. Wiederum kontrastiert die Realität einer enorm umfangreichen Judikatur ${ }^{72}$ mit der Rechtsprechungsmaxime des Gerichtshofs, wonach die primär negativen Konventionsrechte nur ausnahmsweise positive Pflichten begründeten und er diesbezüglich einen „prudent approach ${ }^{\text {“73 }}$ verfolge. ${ }^{74}$ Zwar scheint sich der Gerichtshof bewusst zu sein, dass er sich auf dem Terrain der die Allgemeinheit betreffenden Regelsetzung bewegt, wenn er aus den Konventionsrechten heraus positive Pflichten begründet, denn: „[a] fair balance $[\ldots]$ has to be struck between the general interest of the community and the interests of the individual". ${ }^{75}$ Trotzdem richtet der Gerichtshof immer wieder Gesetzgebungspflichten an die Staaten, namentlich die Pflicht, ein „legal framework“ zum Schutz bestimmter Individualinteressen zu schaffen. ${ }^{76}$ Die Schweiz hatte beispielsweise das zivile Schadenersatzrecht zu revidieren. ${ }^{77}$ Das Beispiel zeigt, dass der Gerichtshof auch Einfluss auf das Zivilrecht der Staaten nimmt. ${ }^{78}$

Eine vierte Gruppe stellen Urteile zum Recht auf freie und faire Wablen dar. ${ }^{79}$ Wenn der Gerichtshof beispielsweise die aus einem komplexen Arrangement der Machtteilung zwischen den wichtigsten ethnischen Gruppen resultierenden Zugangsbeschränkungen zum tripartiten Präsidialamt und dem Haus der Völker (einer parlamentarischen Kammer) von Bosnien und Herzegowina als konventionswidrig beurteilt, ${ }^{80}$ stellt er damit ein zentrales Element der Verfassungsordnung in Frage. Damit nimmt er nicht

70 Kosař/Lixinski, AJIL (2015), 713 (718-719).

71 Kosař, Utrecht L Rev (2017), 112 (123).

72 Lavrysen, Human Rights in a Positive State, 2016, S. 343.

73 EGMR, Urt. v. 10.5.2011 - Nr. 48009/08 - Mosley, Rn. 107; EGMR, Urt. v. 28.4.2009 - Nr.39311/05 - Karakó, Rn. 19.

74 EGMR, Urt. v. 10.3.2020 - Nr. 24816/14 u.a. - Hudorovič, Rn. 139; eingehend Lavrysen, Positive (Fn. 72), S. 214-221.

75 EGMR, Urt. v. 16.7.2013 - Nr. 1562/10 - Remuszko, Rn. 63 (meine Hvhg.).

76 Siehe Lavrysen, Positive (Fn. 72), S. 112-130.

77 EGMR, Urt. v. 11.3.2014 - Nr.52067/10 u.a. - Howald Moor; Bilan d'action, 11.4.2019, 1348th DH mtg, DH-DD(2019)403; Resolution of the Committee of Ministers, 25.9.2019, 1355th DH mtg, CM/ResDH(2019)232.

78 Siehe Zglinksi, YEL (2018), 98 (104-107).

79 Art. 3 Protokoll zur EMRK („Protokoll Nr. 1“), 20.3.1952, (CETS Nr. 009).

80 EGMR, Urt. (GK) v. 22.12.2009 - Nr. 27996/06 u.a., ECHR 2009 - Sejdić and Finci. 
nur in Kauf, dass sein Urteil jahrelang nicht umgesetzt wird ${ }^{81}$ sondern akzeptiert auch die Möglichkeit einer veritablen Verfassungskrise. ${ }^{82} \mathrm{Nach}$ Wheatley entwickelt der Gerichtshof einen konzeptuellen Archetypus des demokratischen Staats, der für alle Mitgliedsstaaten Verbindlichkeit beansprucht. ${ }^{83}$

Schließlich ist die Anerkennung von sozio-ökonomischen Ansprüchen, die aus dem Wirksamkeitsgebot der zivilen und politischen Konventionsrechten fließen, ${ }^{84}$ anzuführen. ${ }^{85}$ Hier bedient sich der Gerichtshof wieder positiver Pflichten. ${ }^{86}$ So gibt es Rechtsprechung zu den Bereichen Gesundheit, Wohnen, Bildung und Arbeit sowie zu finanziellen Sozialleistungen. ${ }^{87}$ Dass es sich dabei um judicial law-making handelt, ist an der politischen Entscheidung erkennbar, die sich in den Vertragsstrukturen des Europarats spiegelt, zivile und politische Rechte einerseits und sozio-ökonomische Rechte andererseits separat zu schützen. ${ }^{8}$ Urteile des Gerichtshofs, die die politische Entscheidung, sozio-ökonomisch Rechte (Eigentumsfreiheit, Recht auf Bildung) ins optionale Protokoll Nr. 1 „auszulagern“, 89 mittels großzügiger Auslegung der zivilen und politischen Rechte umgehen, stoBen denn auch auf Kritik. ${ }^{90}$ Insgesamt stellt Koch fest: „Many of the decisions of the ECtHR have radical consequences for the Contracting Parties $[\ldots]$ with respect to the legislature's liberty to freely adopt and uphold legislation [and] to freely take decisions regarding financial resources". 91

81 Vgl. Resolution of the Committee of Ministers, 2.12.2011, 1128th DH mtg, CM/ ResDH(2011)291; Decision of the Deputies, H46-7, 5.3.2020, 1369th DH mtg, CM/Del/Dec(2020)1369/H46-7.

82 Siehe Abw. M. Bonello.

83 Wheatley, in: Dickinson u. a. (Hrsg.), Critical Perspectives, 2012, S. 153 (172).

84 Grundlegend EGMR, Urt. v. 6.2.1981 - Nr. 6289/73, A-32 - Airey, Rn. 26; EGMR, Urt. v. 13.5.1980 - Nr. 6694/74, A-37 - Artico, Rn. 33.

85 Leijten, Core Socio-Economic Rights, 2018, S. 16.

86 EGMR, Airey (Fn. 84), Rn. 32-33.

87 Koch, Human Rights as Indivisible Rights, 2009, Kap. 5-9.

88 EMRK einerseits, (revidierte) Europäische Sozialcharta, 18.10.1961 bzw. 3.5.1996 (CETS Nr.035 bzw. 163) mit Zusatzprotokoll über Kollektivbeschwerden, 9.11.1995 (CETS Nr. 158), andererseits. Koch, Indivisible (Fn. 87), S. 8.

89 Duranti, Conservative Human Rights Revolution, 2017.

90 Siehe bspw. Abw. M. Keller in EGMR, Urt. v. 12.1.2021 - Nr. 23040/13 - Ryser; Gächter/Meier, HAVE (2016), 480 (482-484).

91 Koch, Indivisible (Fn. 87), S. 255. 


\section{Problemstellung}

Die obigen Beispiele zeigen, dass der Gerichtshof regelmäßig rechtsetzende, also prospektive, an die Allgemeinheit gerichtete Rechte und Pflichten begründende Entscheidungen trifft. Dies kontrastiert mit der prototypischen Funktion der Judikative und ist umgekehrt charakteristisch für die Legislative. ${ }^{92}$ Letztere Trennung liegt in den liberal-demokratischen Grundsätzen aller Autonomie und Gleichheit begründet. ${ }^{93}$ Das liberale Prinzip postuliert, dass allen gleichermaßen erlaubt ist, was nicht allgemein verboten ist ("gleiche Privatautonomie“); das demokratische, dass Freiheitsbeschränkungen in einem Verfahren bestimmt werden, in dem alle gleichermaßen mitbestimmen können („politische Gleichheit“), ${ }^{94}$ weswegen die demokratische der individuellen Selbstbestimmung nicht prinzipiell vorgehen kann. ${ }^{95}$

Nun erlauben Grund- bzw. Konventionsrechte nicht nur individuelle Freiheit, sondern sie schützen gewisse individuelle Freiheitsinteressen, womit Pflichten und Verbote einhergehen: des einen Freiheitsrecht, ist des anderen Verbot, in einer bestimmten Weise zu handeln. Unmittelbar einleuchtend ist das im Falle von „kehrseitigen“ Individualrechtskonflikten. ${ }^{96}$ Sie zeigen, dass auch die individuelle der demokratischen Selbstbestimmung nicht prinzipiell vorgehen kann. ${ }^{97}$ Aber auch wenn der Staat qua Gesamtheit aller Mitglieder der politischen Gemeinschaft verpflichtet ist, bestimmte Ressourcen zur Sicherung eines Grundrechts einzusetzen, impliziert dies ein Verbot, dieselben - per definitionem knappen - Ressourcen zum Schutz anderer Freiheitsinteressen zu verwenden. Notabene gilt diese Logik prinzipiell auch im Bereich klassischer Abwehrrechte: Haftbedingungen im Einklang mit Art. 3 EMRK bedürfen Ressourcen, öffentliche Kundgebungen (Art. 11 EMRK) können Nutzungskonflikte um den öffentlichen Raum verursachen und Eigentumsrechte (Art. 1 Protokoll Nr. 1) bedingen die Errichtung, die Pflege sowie den Schutz und die Durchsetzung entsprechender Rechtsinstitute. All dies setzt funktionierende Systeme der Rechtspflege voraus (Art. 6 EMRK), die ebenfalls Ressourcen beanspruchen. Die Realität, insbesondere in Staaten mit systemischem Reformbedarf und vergleichsweise wenig Mitteln und Kapazitäten, ist mit-

92 Vgl. oben II.1.

93 Beitz, Political Equality, 1989, S. 92-93; Möllers, Branches (Fn. 16), S. 61, 66.

94 Möllers, Branches (Fn. 16), S. 70.

95 Möllers, Branches (Fn. 16), S. 63-65.

96 Vgl. oben II-III.

97 Möllers, Branches (Fn. 16), S. 65-66. 
hin, dass auch im Bereich ziviler und politischer Rechte eine gewisse Priorisierung unumgänglich ist. Die Straßburger Rechtsprechung zeigt denn auch, dass eine scharfe Trennung negativer und positiver Pflichten, die nicht auf Kosten der Wirksamkeit ziviler und politischer Rechte geht, illusorisch ist.

Konventionsrechte bestimmen den Bereich, in dem eine Person, die Rechtsträgerin, über „alle“ bestimmen kann: ${ }^{98}$ wenn sie ihr Recht beansprucht, verpflichtet sie damit die Allgemeinheit. ${ }^{99}$ Mithin betrifft der höchstrechtliche Schutz einer Sphäre individueller Freiheit alle. ${ }^{100}$ Daraus folgt, dass nicht jeder Grundrechtsforderung nachgekommen werden kann und dass prinzipiell in einem demokratischen Verfahren über die geltende Grundrechtskonfiguration zu entscheiden ist. ${ }^{101}$ Weil die Verteilung und Abgrenzung von konventionsrechtlich geschützten Sphären individueller Freiheit alle in ihren Interessen berechtigend und beschränkend betrifft, sollte dies in einem Verfahren geschehen, das alle gleichermaßen mitbestimmen und das die potenziell konkurrierenden Freiheitsinteressen von allen gleichermaßen zu Wort kommen lässt.

Ein kurzer Blick auf die gängigen Institutionen und Verfahren liberaler, rechtsstaatlicher Demokratien zeigt, dass die Legislative diese Ansprüche viel eher erfüllt als die Judikative. ${ }^{102}$ Die Judikative und ihr Verfahren sind darauf ausgerichtet, sich mit Beschwerden über vergangene Verletzungen von geschützten Freiheits- bzw. Interessenssphären, die eine identifizierbare Person aufgrund eines bestimmten Sachverhalts vorbringt, zu beurteilen. Sie verteidigt das liberale Prinzip aller gleicher Privatautonomie im Rahmen der demokratischen Freiheitsbeschränkungen. ${ }^{103}$ Die Legislative und ihr Prozess hingegen sind darauf ausgerichtet, diese Beschränkungen zu bestimmen. Sie hält das demokratische Prinzip aufrecht, wonach alle gleichermaßen mitbestimmen und ihre Freiheitsinteressen einbringen können. ${ }^{104}$

Angesichts des Befunds umfangreichen Straßburger judicial law-making stellt sich die Frage, inwiefern das Verfahren vor dem Gerichtshof, ins-

98 Zur Konzeption individueller Freiheitsrechte als Sozialwahlfunktion Sen, J Pol Eco (1970), 152.

99 Levine, Liberal Democracy, 1981, S. 95, 102.

100 Möllers, Branches (Fn. 16), S. 68.

101 Möllers, Branches (Fn. 16), S. 68-71.

102 Vgl. oben II.1.

103 Möllers, Branches (Fn. 16), S. 67-71, 89-96.

104 Möllers, Branches (Fn. 16), S. 71-76, 84-89; Harlow, MLR (2002), 1 (2). 
besondere in Leitentscheiden, ${ }^{105}$ den institutionellen und prozeduralen Erfordernissen, die das demokratische Prinzip an (quasi-)rechtsetzende Verfahren stellt, gerecht wird.

\section{Problemanalyse}

Was verlangt die Institutionalisierung des demokratischen Prinzips? Erstens müssen alle in einem offenen Verfahren gleichermaßen mitbestimmen können. ${ }^{106}$ Zweiten muss das Verfahren inhaltlich allgemein sein. Drittens bedeutet dies, dass es sich mit der generell-abstrakten Regelung von Sachverhalten beschäftigen muss ${ }^{107}$ und es nicht a priori inhaltlich beschränkt sein darf - alle Freiheitsinteressen müssen eingebracht und wettstreiten können. ${ }^{108}$ Daraus folgt viertens, dass das Verfahren ergebnissoffen zu sein hat. ${ }^{109}$ Fünftens muss das Verfahren in die Zukunft gerichtet sein. Es dient der zukünftigen Orientierung u.a. der individuellen Handlungsfreiheit. ${ }^{110}$ Ein (quasi-)rechtsetzendes Verfahren muss mithin möglichst repräsentativ, partizipativ, transparent und offen sein. Letzteres ist im Sinne größtmöglicher personaler und inhaltlicher Zugangs- bzw. Zulässigkeitsbedingungen zu verstehen. ${ }^{111}$

\section{Repräsentation und Partizipativität}

Das zentrale Problem des judicial law-making liegt in der Überrepräsentation der Interessen der Streitparteien und der Unterrepräsentation der Interessen aller anderen. Der jurisgenerative Prozess fokussiert auf die spezifische Streitsache anstatt auf die allgemeine Politikgestaltung. ${ }^{112}$ Das Problem wird auch nicht dadurch abgeschwächt, dass der Staat im Verfah-

105 Ausgeklammert ist das Verfahren nach Art. 27-28 EMRK.

106 Möllers, Branches (Fn. 16), S. 71, 87-88.

107 Nuanciert Möllers, Branches (Fn. 16), S. 86-87.

108 Möllers, Branches (Fn. 16), S. 72, 75, 85, 87.

109 Möllers, Branches (Fn. 16), S. 76, 85-86.

110 Möllers, Branches (Fn. 16), S. 75-76.

111 Vgl. Anagnostou, in: Anagnostou (Hrsg.), Rights and Courts, 2014, S. 1 (16-20); Andersen, Out of the Closets, 2005, S. 6-14.

112 Bellamy, Political (Fn. 51), S. 246; Miller, in: Webber u. a. (Hrsg.), Legislated Rights, 2018, S. 181 (196-197); Möllers, in: Jestaedt u. a. (Hrsg.), Das entgrenzte Gericht, 2011 (316-318); von Bogdandy/Venzke, LJIL (2013), 49 (59-63); von Bogdandy/Venzke, Beyond Dispute (Fn. 14), S. 24-26. 
ren vor dem Gerichtshof als Streitpartei (i.d.R. auch nur einer von $46^{113}$ ) auftritt, denn dort wird er in aller Regel von einem spezialisierten agent de gouvernement der wenig repräsentativen Exekutive vertreten. ${ }^{114}$ Internationales Richterrecht schwächt die repräsentative Legislative innerhalb der nationalen trias politica. ${ }^{115}$

Das Problem ist sodann auch eines der Partizipation. Nur die Streitparteien partizipieren am judicial law-making. Hingegen steht der legislative Prozess grundsätzlich allen offen. Dieses Problem mildern Drittstaatsinterventionen ${ }^{116}$ und zivilgesellschaftliche amici curiae ab. ${ }^{117}$ Kritisch betrachtet füllen letztere aber ein Machtvakuum geschickt mit ihren Partikularinteressen aus. ${ }^{118}$

\section{Zugangs- und Zulässigkeitsbedingungen}

Gerichtsverfahren ermächtigen nicht notwendigerweise diejenigen, die im politischen Prozess kaum zu Wort kommen. Ähnliche Ressourcen dürften über die juristische und die politische Schlagkraft entscheiden. ${ }^{119}$ Diesbezüglich ist in Straßburg ein Problem besonders markant: Die Rechtshilfe leistet nur einen bescheidenen Beitrag ${ }^{120}$ und dies erst ab einem späten Verfahrensstadium. ${ }^{121}$ Etwa 87 Prozent der Beschwerden scheitern vorher aufgrund formaler Erfordernisse oder der Zulässigkeitskriterien. ${ }^{122}$

113 Vgl. Helfer/Voeten, IO (2014), 77 (78).

114 Rule 35 RC; Jenart/Leloup, EuConst (2019), 247 (255-256).

115 Sajó/Giuliano, in: Klabbers/Palombella (Hrsg.), Challenge of Inter-Legality, 2019, S. 230 (235-236); von Bernstorff, in: Mendes/Venzke (Hrsg.), Allocating Authority, 2018, S. 99 (109); vgl. auch Issacharoff, U Chi L Rev (2018), 485 (499-501).

116 Bürli, Third-Party Interventions, 2017, Kap. 3.

117 Art. 36(2) EMRK; Bürli, Third-Party (Fn. 116), S. 20-22.

118 Sajó/Giuliano, Perils (Fn. 115), S. 236.

119 Bellamy, Political (Fn. 51), S. 42; Tushnet, GLJ (2013), 2249 (2255); bspw. EGMR, Urt. (GK) v. 25.5.2021- Nr. 58170/13 u.a. - Big Brother Watch.

120 Leach, Taking a Case to the ECtHR, 4. Aufl., 2017, App. 8.

121 Rule 105 RC.

122 Rule 47 RC. Über 7`000 Beschwerden wurden auf dieser Grundlage abgewiesen, was ca. $18 \%$ der $41^{6} 700$ den Spruchkörpern zugeteilten Beschwerden entspricht. Ähnlich viele Beschwerden wurden aus anderen administrativen Gründen zurückgewiesen. Nur 7‘681 Beschwerden wurden kommuniziert und erreichten das Stadium, wo Rechtshilfe verfügbar wird. EGMR, Analysis of Statistics 2020, 2021, S. 4; siehe auch Bianku/Kempees, in: Baetens (Hrsg.), Legitimacy of Unseen Actors, 2019, S. 108 (111-113); Schädler, Re-Designing the Admissibility Model, 2019, S. 14. 
Die Zulässigkeitspraxis, namentlich die große Zahl an Beschwerden, die als „manifestly ill-founded“ und somit gestützt auf eine inhaltliche Vorprüfung abgewiesen werden, ${ }^{123}$ wird immer wieder kritisiert. Mehr als die Hälfte aller Unzulässigkeitsentscheidungen dürfte primär auf dieses Kriterium abstellen. ${ }^{124}$ Die Straßburger Praxis gleicht einem Certiorari-System, das Zulässigkeitsentscheidungen dem richterlichen Ermessen anheimstellt. ${ }^{125}$ In diesem Sinn garantiert das Beschwerderecht kein Recht auf Zugang zum Gerichtshof. ${ }^{126}$ Hinzu kommt, dass grösstenteils non-judicial rapporteurs die Fallselektion übernehmen: ${ }^{127}$ „legal staff sets the judicial agenda". ${ }^{228}$

Auch für NGOs ist der Zugang zum Gerichtshof schwierig. Etwa die Hälfte aller amici curiae wird abgelehnt, und zwar auch in wegweisenden, kontroversen Fällen. ${ }^{129}$ Die Praxis ist intransparent. ${ }^{130}$ Einen Anspruch auf Stellungnahme gibt es nicht. ${ }^{131}$ Man könnte fürchten, dass wenige spezialisierte Organisationen einen Großteil aller Stellungnahmen auf sich vereinigen, ${ }^{132}$ doch scheint dies nicht ausgeprägt zu sein. ${ }^{133}$

In Leiturteilen kann Akteuren der strategischen Prozessführung bzw. -unterstützung - der "continuation of politics by other means"134 - eine wichtige Rolle zukommen. Spezialisierte NGOs könnten hinter einem erheblichen Teil aller Leiturteile stehen ${ }^{135}$ und angesichts der selektiven

123 Art.35(3)(a) EMRK; EGMR, Practical Guide on Admissibility Criteria, 30.4.2020, Rn. 297; bspw. EGMR, Ent. v. 7.12.2004 - Nr. 71074/01, ECHR 2004XII - Mentzen.

124 EGMR, Facts About Admissibility, 2017, <https://cutt.ly/NkMwwcJ>, abgerufen am: 31.3.2021; Tickell, Is the European Court of Human Rights Obsessively Interventionist?, <https://cutt.ly/HkNZCYK>, abgerufen am: 31.3.2021.

125 Cameron, in: Føllesdal/Peters/Ulfstein (Hrsg.), Constituting Europe, 2013, S. 25 (57).

126 Schädler, Admissibility (Fn. 122), S. 19 m. H.

127 Siehe Bianku/Kempees, ECtHR (Fn. 122), S. 111-113.

128 Schädler, Admissibility (Fn. 122), S. 199.

129 Bürli, Third-Party (Fn. 116), S. 116.

130 Bürli, Third-Party (Fn. 116), S. 115.

131 Rule 44 RC.

132 Vgl. Bellamy, Political (Fn. 51), S. 245; Føllesdal, NJHR (2009), 289 (302).

133 Bürli, Third-Party (Fn. 116), S. 114; van den Eynde, NQHR (2013), 271 (285).

134 O'Neill, Strategic (Fn. 134), S. 506-508; siehe dazu auch den Beitrag von Strobel in diesem Band, Strategische Prozessführung - Potentiale und Risiken transnationaler zivilgesellschaftlicher Zuflucht zum Recht.

135 Vgl. Anagnostou, Minorities (Fn. 111), S. 10; Anagnostou, in: Anagnostou (Hrsg.), Rights and Courts, 2014, S. 205 (211); Lewis, EHRLR (2011), 700 (706-713); O'Neill, ERA Forum (2015), 495 (506-508). 
Urteilspraxis des Gerichtshofs als „Türhüter“ fungieren. Eine Beurteilung ist jedoch schwierig, weil NGOs oft hinter den Kulissen operieren. ${ }^{136}$ Problematisch ist es auch, wenn NGOs „strategisch“ ein politikänderndes Urteil erringen, das gar nicht alle vermeintlich Begünstigten begrüßen. ${ }^{137}$

\section{Offenheit}

Der Gesetzgebungsprozess steht grundsätzlich allen Anliegen offen. (Kompromiss-) Lösungen werden sachübergreifend erarbeitet. ${ }^{138} \mathrm{Im}$ Gerichtsverfahren ist das anders. Erstens ist es inhaltlich auf die Streitsache limitiert. ${ }^{139}$ Zweitens kann nur dann auf vergangene Entscheidungen zurückgekommen werden, wenn eine entsprechende Beschwerde eingereicht wird. ${ }^{140}$ Drittens ist der politische Prozess argumentativ offener als der judikative. Ersterer erlaubt grundsätzlich jede Argumentation, während zweiterer bestimmten semantischen und argumentativen Mustern folgt. ${ }^{141}$ Trotz lobenswerter Öffentlichkeitsarbeit ${ }^{142}$ haben nur Spezialisten den Überblick über die immense Praxis des Gerichtshofs. ${ }^{143}$ Der Kasuismus, die Abweichungen zwischen der Rechtsprechung verschiedener Sektionen ${ }^{144}$ und die sich ständig ändernde Konventionsauslegung ${ }^{145}$ bevorteilt Experten in der Prozessführung vor dem Gerichtshof. ${ }^{146}$

136 Hodson, in: Anagnostou (Hrsg.), Rights and Courts, 2014, S. 181 (184).

137 Zu EGMR, Urt. (GK) v. 13.11.2007 - Nr. 57325/00 - D.H., Helmrich, in: Graser/ Helmrich (Hrsg.), Strategic Litigation, 2019, S. 115 (137) m. w. H.

138 Eingehend Bellamy, Liberalism and Pluralism, 1999, Kap. 4-5; siehe bspw. auch EGMR, Urt. (GK) v. 17.5.2016 - Nr. 42461/13 u.a., ECHR 2016 - Karácsony, Rn. 141.

139 Bspw. EGMR, Urt. (GK) v. 5.9.2017 - Nr. 78117/13 - Fábián, Rn. 81.

140 Art. 34 EMRK; EGMR, Urt. (GK) v. 4.12.2015 - Nr. 47143/06, ECHR 2015 - Roman Zakharov, Rn. 164 (actiones populares unzulässig); allg. Raz, Ind LJ (1995), 27 (42).

141 Miller, Majoritarianism (Fn. 112), S. 195; Venzke, How Interpretation Makes International Law, 2012, S. 46-57; Waldron, Yale LJ (2006), 1346 (1380-1386).

$142<$ https://www.echr.coe.int $>$.

1432020 erließen die Kammern und die Große Kammer 391 Urteile. Siehe EGMR, Statistics 2020 (Fn. 122), S. 5.

144 Organisationseinheiten aus denen sich die Spruchkörper bilden.

145 Siehe Árnardóttir, NJHR (2015), 220 (240-241); Cameron, Court (Fn. 125), S. 5354; Gerards, HRLR (2018), 495 (512); Kratochvíl, NQHR (2011), 324 (352); Touzé, in: Sudre (Hrsg.), Principe de subsidiarité, 2014, S. 59 (76-77).

146 Allg. dazu Waldron, Emory LJ (2009), 675 (693-697); vgl. auch Tomuschat, RdC (1999), 9 (28). 


\section{Zwischenfazit}

Der Gerichtshof ist, wie für die Judikative üblich, auf die Streitbeilegung im Zweiparteienverfahren ausgerichtet. In wegweisenden Verfahren sind die Streitparteien über- und die übrigen Betroffenen unterrepräsentiert. Die Zulässigkeits-, Zugangs- und Partizipationsmöglichkeiten sind rigide und ihre Handhabung teils opportunistisch oder gar scheinbar willkürlich. Auch die beschränkte inhaltliche Offenheit des Verfahrens vor dem Gerichtshof zeugt von seinem Charakter als spezialisiertes Forum der bilateralen Streitbeilegung. Anzufügen ist, dass der EGMR als internationaler Gerichtshof anders als nationale Höchstgerichte ${ }^{147}$ kaum in eine ausgebildete politische Demokratie und ihre legitimitätstiftenden Strukturen öffentlicher Meinungsbildung eingebunden ist. ${ }^{148}$ Insgesamt genügt der Gerichtshof den institutionellen und prozeduralen Erfordernissen, die das demokratische Prinzip an (quasi-)rechtsetzende Verfahren stellt, somit kaum.

\section{Korrektive}

Angesichts der identifizierten Probleme stellt sich die Frage nach möglichen Korrektiven. Der vorliegende Beitrag muss sich auf einige recht einfach umzusetzende und relativ unkontroverse Maßnahmen beschränken und kann weitreichenden, innovativen Ideen nicht gerecht werden.

Amici curiae tragen dazu bei, dem Gerichtshof in Leitverfahren ein breites Spektrum gesellschaftlicher Interessen wirksam ${ }^{149}$ zur Kenntnis zu bringen. ${ }^{150} \mathrm{Um}$ größere Transparenz herzustellen, sollte der Gerichtshof über die angenommenen und abgelehnten amici curiae begründet informieren. Die Praxis muss nachvollziehbar und einheitlich sein sowie Orientierung für die Zukunft bieten. ${ }^{151}$ Zudem ist transparent zu garantieren, dass inhaltlich möglichst vielfältige Positionen ausgewogen zu Wort kom-

147 Vgl. bspw. Bailey/Maltzmann, Constrained Court, 2011, Kap. 6-7.

148 Bellamy, ICON (2011), 86 (96); Langvatn, Ethics \& Intl Aff (2016), 355 (366367).

149 Vgl. Busch/Reinhardt, World Politics (2006), 446.

150 Bürli, Third-Party (Fn. 116), S. 21-22.

151 Vgl. Bürli, Third-Party (Fn. 116), S. 116. 
men. ${ }^{152}$ Legislativmitglieder der Konventionsstaaten können und sollten sich ebenfalls einbringen. ${ }^{153}$

Es sollte auch Transparenz darüber herrschen, wer eine Beschwerde mit Ressourcen unterstützt und welche Interessenbindungen bestehen. Aufgrund vieler Verbindungen zwischen Gerichtsmitgliedern und NGOs würde dies die richterliche Unabhängigkeit stärken. ${ }^{154}$ Die Ausstandsregeln ${ }^{155}$ sind streng anzuwenden.

Rechtshilfe sollte - auch im Interesse ausgewogener Rechtsprechung ${ }^{156}$ - ausreichend gewährt werden. Statistisch ist die Zulässigkeit einer Beschwerde die größte Hürde. Dementsprechend sollten bereits in diesem Stadium alle, die Unterstützung brauchen, qualifizierte Hilfestellung erhalten. ${ }^{157}$ Unverständlich ist, dass die Rechtshilfe nur standardisierte Beiträge für die Prozesskosten leistet. ${ }^{158}$ Insbesondere Anwaltskosten dürften enormen Unterschieden zwischen den Konventionsstaaten unterliegen, und selbst bzw. gerade in den reichsten Ländern gibt es Menschen, die sich eine anwaltliche Vertretung auch mit dem Beitrag der Rechtshilfe nicht leisten können. ${ }^{159}$ Wünschenswert wäre auch eine öffentliche Liste mit "pro bono lawyers“. ${ }^{60}$

Weiter müsste der Gerichtshof seine Zulässigkeitspraxis überdenken. Schädler diskutiert dies eingehend. Zustimmung verdienen vier Punkte. Erstens sollten die Anforderungen für Beschwerden ohne anwaltliche Vertretung gelockert werden. ${ }^{161}$ Zweitens sind die materiellen Zulässigkeitskriterien zu objektivieren. ${ }^{162}$ Drittens ist das Kriterium „manifestly ill-founded" nur auf Beschwerden anzuwenden, die tatsächlich manifest unbegründet sind und nicht sowieso an einer spezifischeren Zulässigkeits-

152 Vgl. Bürli, Third-Party (Fn. 116), S. 122.

153 EGMR, Urt. (GK) v. 18.3.2011 - Nr. 30814/06, ECHR 2011 - Lautsi, Rn. 56; Bürli, Third-Party (Fn. 116), S. 126.

154 Villiger, Handbuch (Fn. 23), Rn. 998; siehe auch Harlow, MLR (2002), 1 (13).

155 Rule 28 RC.

156 Butler, ICLQ (2000), 360 (363) (Anm. 10).

157 Vgl. aber Rule 36 RC, wonach das grundsätzliche Vertretungsgebot bzw. -erfordernis erst später einsetzt.

158 Hier wie anderswo rühren die Probleme u.a. von den äußerst knappen Mitteln her, die die Staaten zur Verfügung stellen. Siehe auch Schädler, Admissibility (Fn. 122), S. 236.

159 Vgl. 106(b) RC.

160 Nach Butler, Legal Aid (Fn. 156), S. 368, gab es das früher.

161 Vgl. Schädler, Admissibility (Fn. 122), S. 239-250.

162 Vgl. Schädler, Admissibility (Fn. 122), S. 274-282. 
bedingung scheitern. ${ }^{163}$ Zustimmung verdient schließlich die ausnahmsweise Lockerung der Zulässigkeitskriterien im Fall von Beschwerden, die die Nichterfüllung eines Kriteriums entschuldbar erscheinen lassen, sowie im Fall systemischer Probleme. ${ }^{164}$

Was die Offenheit des Verfahrens anbelangt, könnte die Rechtsprechung der Großen Kammer Besserung bringen. Wenn ein umstrittenes Kammer-Urteil nochmals verhandelt wird, führt das immerhin zur zweimaligen Deliberation der Kontroverse. Diese Überlegung spricht gegen die Abgabe, ${ }^{165}$ aber für die Verweisung ${ }^{166}$ von wegweisenden Fällen an die Große Kammer. Beschwerden, die vor der Großen Kammer hängig sind, bekommen zudem viel Aufmerksamkeit, was regelmäßig zu mehr Interventionen seitens amici curiae und Drittstaaten führt. ${ }^{167}$

Problematisch ist die intransparente und mitunter willkürlich anmutende Praxis des Gerichtshofs, die unklar lässt, wann eine Beschwerde an die Große Kammer verwiesen wird. ${ }^{168}$ Es wäre prüfenswert, ob man eine Gruppe von Konventionsstaaten, die Parlamentarische Versammlung und/oder den Menschenrechtskommissar dazu ermächtigen könnte, die Verweisung einer Beschwerde an die Große Kammer zu verlangen. Damit könnte eine Frage, die unberechtigterweise unter dem Radar blieb, einer eingehenderen Prüfung unter Einbezug weiterer Kreise zugänglich gemacht werden. ${ }^{169}$

Der Gerichtshof ist und bleibt eine judikative Institution. Doch werfen die Dimensionen seines judicial law-making gewaltenteilungstheoretische Probleme auf, die es zu minimieren gilt. Leider ist der Gerichtshof teils unwillig, teils unfähig seine Verfahrensordnung entsprechend zu ändern. Insbesondere bleibt seine Praxis hinsichtlich amici curiae und Verweisungen an die Große Kammer trotz Kritik unverändert intransparent und dem Ermessen einzelner Gerichtsmitglieder überlassen. Auch der Aufforderung, nicht am Verfahren beteiligten Staaten eine Möglichkeit zu geben, dem Wunsch nach einem Urteil der Großen Kammer Ausdruck zu verleihen, hat er bislang soweit ersichtlich nicht Folge geleistet.

163 Vgl. Schädler, Admissibility (Fn. 122), S. 282-293.

164 Vgl. Schädler, Admissibility (Fn. 122), S. 294-299.

165 Art. 30 EMRK; aber Art. 3 P-15; dazu Expl. Rep. P-15 (Fn. 8), Rn. 16-20.

166 Art. 43 EMRK.

167 Bürli, Third-Party (Fn. 116), S. 113-114.

168 Rule 73 RC; EGMR, Urt. v. 12.1.2021 - Nr. 23040/13 - Ryser, Rn. 10-11 (Abw. M. Keller). Rainey/McCormick/Ovey, ECHR, 8. Aufl., 2021, S. 19.

169 Vgl. Kopenhagen Deklaration (Fn. 7), Rn. 38-40. 
Nebst den diskutierten institutionell-prozeduralen Korrektiven steht die Rückbindung der evolutiven Konventionsauslegung an die politisch-rechtlichen Entwicklungen in den Staaten als Mittel der demokratischen Rückkopplung des Straßburger judicial law-making zur Debatte. Dabei geht es jedoch um die Auslegungsmethodik und das Rollenverständnis des Gerichtshofs - Fragen, die hier nicht behandelt werden können. ${ }^{170}$

\section{Schlussbemerkungen}

Der Gerichtshof kann und soll nicht zu einem Forum der Rechtsetzung werden, das gleichermaßen demokratisch legitimiert ist wie funktionierende Parlamente liberaler Demokratien. ${ }^{171}$ Weil die Glaubwürdigkeit einiger Konventionsstaaten als liberale Demokratien schwach und keine liberale Demokratie unfehlbar ist, spielt der Gerichtshof trotz der gewaltenteilungstheoretischen Probleme, die sein judicial law-making aufwirft, eine legitime demokratische Rolle. ${ }^{172}$ Denn erstens trifft funktionierende Demokratien eine „duty of justice“, ${ }^{173}$ Menschenrechtsgerichte, die Grundrechte (auch) in anderen Ländern fördern, zu unterstützen und ihnen beizutreten, um „Gruppendruck“ zu erzeugen. ${ }^{174}$ Zweitens verletzt auch der demokratischste Staat mitunter Menschenrechte. Der externe Blick des Gerichtshofs beugt insbesondere der innerstaatlichen Verletzung der Rechte von Minderheiten und von strukturell schwachen Gruppen vor. ${ }^{175}$ Drittens dürfte der Gerichtshof das Vertrauensverhältnis zwischen einem Mitgliedstaat und den seiner öffentlichen Gewalt Unterworfenen generell stärken, weil er Missbrauch unwahrscheinlicher macht. ${ }^{176}$ Dies - und damit schließt sich der Kreis gewissermaßen - setzt allerdings voraus, dass

170 Dazu Dzehtsiarou, European Consensus, 2015, Kap. 5-7.

171 Oben III; Harlow, MLR (2002), 1 (11); Tomkins, Republican Constitution, 2005, S. 3 .

172 Føllesdal, in: Føllesdal/Schaffer/Ulfstein (Hrsg.), Legitimacy of International Human Rights Regimes, 2013, S. 272 (279-284) m. w. H; Føllesdal, Theo Inq L (2013), 339.

173 Rawls, Theory of Justice, 1999, $\$ \$ 19,51$.

174 Føllesdal, Much Ado (Fn. 172), S. 281-282; Simmons, Mobilizing for Human Rights, 2009, S. 13.

175 Føllesdal, Much Ado (Fn. 172), S. 282; siehe auch Anagnostou, Minorities (Fn. 111), S. 3 m. w. H.

176 Føllesdal, Much Ado (Fn. 172), S. 283; siehe auch Buchanan, Pol, Phil \& Econ (2011), 5 (6). 
der Gerichtshof als legitim betrachtet wird. ${ }^{177}$ Wird er das nicht, beispielsweise aufgrund übertriebenem „rechtsetzendem Aktivismus“, droht er zur Zielscheibe von nationalen Populisten zu werden, deren Programm er mitunter zu befeuern riskiert, wenn er Entscheidungen der Mitgliedstaaten in kontroverser Weise in Frage stellt. ${ }^{178}$

Es wäre mithin falsch, jede gewaltenteilungstheoretische Kritik am judicial law-making des Gerichtshofs als gegen die Institution gerichtet zu sehen. Vielmehr kann sie ein Bemühen sein, zu erörtern, welche Rolle dem Gerichtshof im Spannungsfeld zwischen wertvoller internationaler gerichtlicher Kontrolle und Verlangen nach nationaler demokratisch-politischer Selbstbestimmung legitimerweise zukommt. 